\title{
The Speed of Epidemic Waves in a One-Dimensional Lattice of SIR Models
}

\author{
Igor Sazonov $^{a 1}$, Mark Kelbert ${ }^{b}$ and Michael B. Gravenor ${ }^{c}$ \\ ${ }^{a}$ School of Engineering, Swansea University, Singleton Park, SA2 8PP, U.K. \\ ${ }^{b}$ Department of Mathematics, Swansea University \\ ${ }^{b}$ Institute of Life Science, School of Medicine, Swansea University
}

\begin{abstract}
A one-dimensional lattice of SIR (susceptible/infected/removed) epidemic centres is considered numerically and analytically. The limiting solutions describing the behaviour of the standard SIR model with a small number of initially infected individuals are derived, and expressions found for the duration of an outbreak. We study a model for a weakly mixed population distributed between the interacting centres. The centres are modelled as SIR nodes with interaction between sites determined by a diffusion-type migration process. Under the assumption of fast migration, a one-dimensional lattice of SIR nodes is studied numerically with deterministic and random coupling, and travelling wave-like solutions are found in both cases. For weak coupling, the main part of the travelling wave is well approximated by the limiting SIR solution. Explicit formulae are found for the speed of the travelling waves and compared with results of numerical simulation. Approximate formulae for the epidemic propagation speed are also derived when coupling coefficients are randomly distributed, they allow us to estimate how the average speed in random media is slowed down.
\end{abstract}

Key words: spatial epidemic models; dynamic systems; travelling waves

AMS subject classification: 92D30, 91D25

\section{Introduction}

Mathematical models have been long used to study the epidemiology of a wide range of infectious diseases [1]. The classical SIR (susceptible/infected/removed) model is one of the cornerstones of

\footnotetext{
${ }^{1}$ Corresponding author. E-mail: i.sazonov@swansea.ac.uk
} 
mathematical epidemiology, describing the dynamics of infection in a single population $[18,5]$. The analysis of infection spread through linked systems of populations such as urban centres is of great importance [20] and attracts considerable interest, in particular for planning the response to emerging pandemic diseases. An excellent review of a huge literature on spatial epidemic models can be found in [15].

The numerical simulation of spatial systems gives insight into different scenarios of epidemic spread and can be used to help plan actual interventions. However, the inclusion of large numbers (potentially many thousands) of interacting nodes can be costly in terms of integrating the SIR processes, and simplified models (e.g., with the epidemic processes greatly simplified within the nodes) are widely used. For example, during the recent foot and mouth disease outbreak in the UK [9] 'real time' modelling of the spatial spread of the epidemic was performed, on the network of almost all sheep/cattle/pig livestock premises in the country. Following this experience, recommendations were made for the construction, in advance, of well defined and understood quantitative models as part of attempts to prepare for future outbreaks of emerging diseases.

A well-developed simulation technique is based on passing to the continuous media $[18,8$, $14,6,3]$. Usually this limit leads to PDE of the reaction-diffusion type. A well-known feature is the existence of the so-called travelling wave solutions, that preserve speed and shape. Analogous patterns are systematically observed in epidemic data sets [6]. However, situations are possible when the continuous limit is not applicable, although similar patters can occur. Analysis of the discrete nature of spatial interactions is one our work goals.

Many recent publications focus on the spatial aspect, and the propagation of disease (or computer viruses or rumours) in networks with different topology attracts a lot of attention (see review [10] and papers cited therein, in particular, the 'Small World' network [17]). Usually, these models (i.e. the contact processes) apply a simplified description of the epidemic within a single node (down to the simplest binary model: infected or not). As a step towards including more detailed epidemic dynamics into the nodes of these lattice-type spatial models, it is important to consider a system of locally interacting SIR models and to study their 'global' behaviour.

Here, we consider a network with the simplest topology - a 1D lattice with a large number of interacting nodes. The dynamics within each node is described by a standard SIR model, with additional terms accounting for the interaction between nodes. More precisely, we use an approximation of weak coupling and fast migration to obtain a relatively simple and explicit description of epidemic propagation. Using an approximation of weak coupling, we assume that the interaction is due to migration of a relatively small proportion of the population (including those infective) between nodes, and the interaction is proportional to the small share of time this population spends in the neighbouring nodes. The situation is therefore most applicable when populations are concentrated mainly in their own communities, and the spread of an epidemic has a distinctive time delay caused by a lack of strong mixing in the overall population.

We establish the existence of travelling wave type solutions in such systems, under deterministic and random coupling and determine its speed of propagation. It is shown that in an interesting parameter range it is impossible to approximate the finite differences by derivatives, i.e. to pass to reaction-diffusion equations, and a discrete spatial approach is essential for the analysis.

The paper is organized as follows. In Section 2, the limiting solutions of a single SIR model 
are introduced. They provide a good approximation for an outbreak triggered by a small number of initial infectives, and provide the base for describing travelling waves in the lattice of SIR nodes in Section 4. In Section 3 the migration model for spread between nodes is discussed, and equations of interacting SIR models are described. In Section 4, travelling waves in a 1D lattice of interacting identical SIR models are investigated. In Section 5, the study is extended for a lattice with random coupling. In Section 6, we study the limits of applicability of the approximation used in analysis, estimate the possible errors and consider the continuous case. Finally, in Section 7, we draw our conclusions, and discuss the possible extensions of the model.

\section{Limiting solution of the SIR model}

In the classical SIR model, the numbers of susceptibles $S$, infectives $I$ and removed (immune/dead due to disease/vaccinated) $R$ evolve in accordance with the equations

$$
\frac{d}{d t} S=-\beta S I, \quad \frac{d}{d t} I=\beta S I-\alpha I, \quad \frac{d}{d t} R=\alpha I
$$

where $\beta$ is the infection rate, $\alpha$ the recovery rate (e.g. $[18,5])$. Adding these equations and integrating the sum, we obtain $S+I+R=$ const $=N$ where $N$ is the population number. The typical evolutionary problem is to integrate (2.1) if the initial number of infectives $I_{0}$ is set at $t=t_{0}$.

We write equations (2.1) in the dimensionless form

$$
\begin{aligned}
\frac{d}{d \tau} s & =-\rho s i \\
\frac{d}{d \tau} i & =(\rho s-1) i
\end{aligned}
$$

where $s=S / N, i=I / N$, are, respectively, the shares of susceptibles and infectives, and $\tau=\alpha t$ is the dimensionless time. Here $\rho=(\beta / \alpha) N$ is called the basic reproduction number usually denoted by $\mathrm{R}_{0}[18,1,7]$. The differential equation for $r$ can be omitted as $r=1-s-i$. The initial conditions in these variables can be written as

$$
i\left(\tau_{0}\right)=i_{0}, \quad s_{0}:=s\left(\tau_{0}\right)=1-i_{0} .
$$

The initial number of infectives grows if $\rho s_{0}>1$ [5] and reaches its maximal value [5]

$$
i_{\max }=1-\rho^{-1}+\rho^{-1} \ln \left(\rho^{-1} s_{0}^{-1}\right)
$$

at an instant which we denote as $\tau_{\max }$. Then the outbreak time can be defined as [5]

$$
\tau_{\text {outb }}:=\tau_{\max }-\tau_{0} .
$$

In Figure 1a, the solutions $i(\tau)$ are plotted for $\rho=4$ and various values of $i_{0}$. Initial instants $\tau_{0}$ are chosen to achieve the maximum $i_{\max }$ at the same instant. Observe that as $i_{0}$ decreases, the 


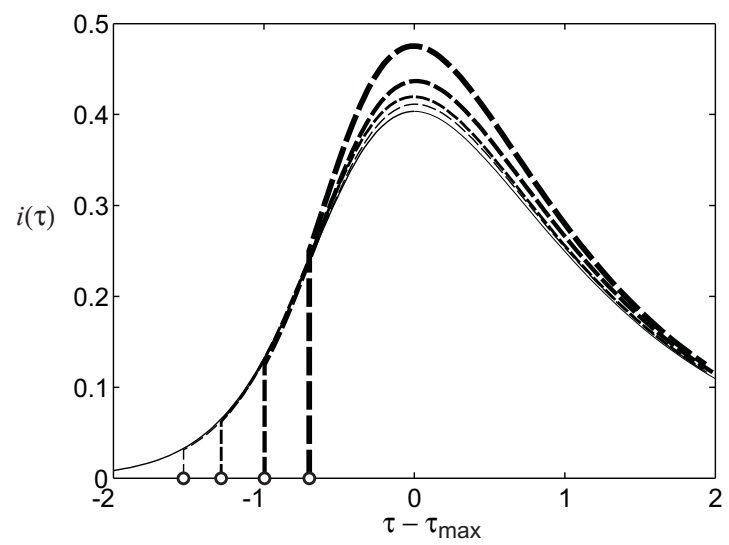

a

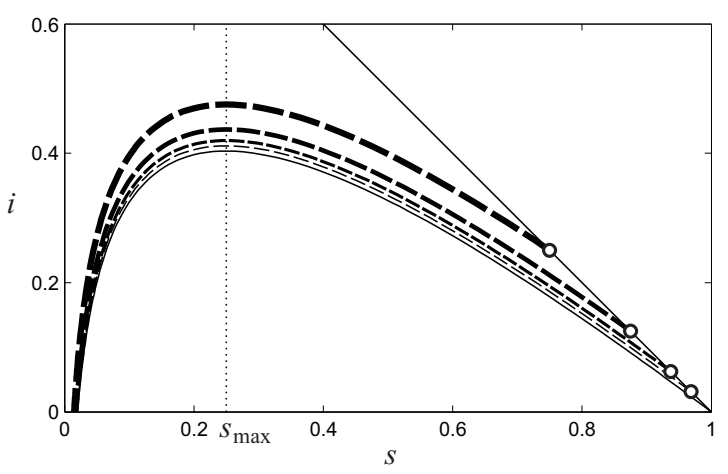

b

Figure 1: Solutions $i(\tau)$ (a) and $i(s)$ (b) for $i_{0}=1 / 4,1 / 8,1 / 16,1 / 32$ and $\rho=4$ (dashed curves: the smaller $i_{0}$ the thinner is the curve). The thin solid curve indicates the limiting solution.

solutions tend to an unique limiting solution $i^{\lim }(\tau)$ starting from the point $i=0, s=1$ at the instant $\tau=-\infty$. On the phase diagram $\{s, i\}$, the limiting solution $i^{\lim }(s)$ is the lower boundary of all realistic trajectories (Figure 1b).

The limiting solutions $i^{\lim }(\tau)$ are shift invariant with respect to $\tau$, and from now on we set $\tau_{\max }=0$. They then form a one parameter family plotted in Figure 2.

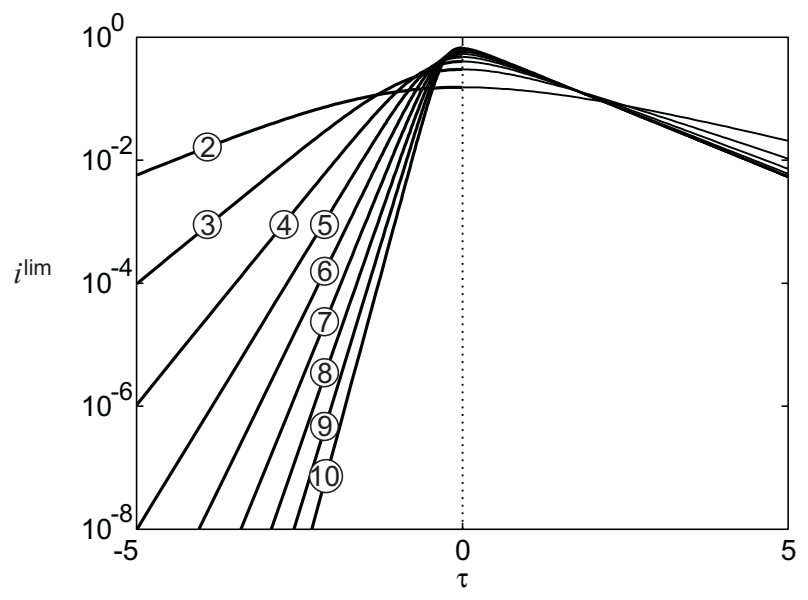

Figure 2: Limiting solutions $i(\tau)$ for $\rho=2,3, \ldots, 10$ in the logarithmic scale. Values of $\rho$ are indicated in circles.

The limiting solutions have simple asymptotic behaviors

$$
\begin{array}{ll}
i^{\lim }=A_{0} e^{\lambda_{0} \tau} & \text { if } \tau \rightarrow-\infty \\
i^{\lim }=A_{\infty} e^{\lambda_{\infty} \tau} & \text { if } \tau \rightarrow+\infty
\end{array}
$$


where $A_{0}$ and $A_{\infty}$ are some constants; $\lambda_{0}=\rho-1>0, \lambda_{\infty}=-1+\rho s_{\infty}^{\lim }<0$ are the growth/decay rates. Here

$$
s_{\infty}^{\lim }:=\lim _{\tau \rightarrow \infty} s^{\lim }(\tau)=-\frac{1}{\rho} W_{0}\left(-\rho e^{-\rho}\right)
$$

where $W_{k}(z)$ is the $k$ th branch of the Lambert function [4] (see Appendix for details).

Any solution started at $\tau_{0}$ with small enough $i_{0}$ can be approximated by the limiting curve

$$
i\left(\tau ; \rho, i_{0}, \tau_{0}\right) \approx i^{\lim }\left(\tau-\tau_{0}-\tau_{\text {outb }} ; \rho\right) .
$$

The maximal discrepancy between usual and limiting solutions occurs in the vicinity of maximum of $i$ and approximately equals

$$
\max \left|i-i^{\lim }\right| \approx i_{\max }-i_{\max }^{\lim }=-\frac{\ln \left(s_{0}\right)-1}{\rho} \approx \frac{i_{0}}{\rho} .
$$

To apply approximation (2.9) it is necessary to calculate $\tau_{\text {outb }}$ for given $\rho$ and $i_{0}$. An integral formula for the outbreak time is

$$
\tau_{\text {outb }}=\int_{s_{\max }}^{s_{0}} \frac{d s}{\rho\left(s-s^{2}\right)+s \ln \left(s / s_{0}\right)}
$$

where

$$
s_{\max }:=s\left(\tau_{\max }\right)=\rho^{-1} .
$$

This value can be easily obtained by setting $d i / d \tau=0$ in (2.3); substituting (2.11) into (A1) we obtain (2.5).

When $i_{0} \rightarrow 0$, non-decaying asymptotic terms of (2.10) are

$$
\tau_{\text {outb }} \cong \frac{1}{\lambda_{0}} \ln \frac{1}{i_{0}}+C+O\left(i_{0}\right)
$$

where $C=C(\rho)$ is independent of $i_{0}$. The proof, and the integral formula for $C(\rho)$ are given in the Appendix. The constant $C$ is related to the constant $A_{0}$ in (2.6)

$$
C=\lambda_{0}^{-1} \ln A_{0} \text {. }
$$

\section{Interacting SIR processes}

Consider the migration process between two interacting identical centres. Then the rate of change of infectives/susceptibles will be determined by migration as well, and additional terms appear in the equations

$$
\begin{aligned}
\frac{d}{d \tau} s_{1} & =-\rho s_{1} i_{1} \quad+\frac{d}{d \tau} s_{2 \rightarrow 1}-\frac{d}{d \tau} s_{1 \rightarrow 2} \\
\frac{d}{d \tau} i_{1} & =\rho s_{1} i_{1}-i_{1}+\frac{d}{d \tau} i_{2 \rightarrow 1}-\frac{d}{d \tau} i_{1 \rightarrow 2} \\
\frac{d}{d \tau} s_{2} & =-\rho s_{2} i_{2} \quad+\frac{d}{d \tau} s_{1 \rightarrow 2}-\frac{d}{d \tau} s_{2 \rightarrow 1} \\
\frac{d}{d \tau} i_{2} & =\rho s_{2} i_{2}-i_{2}+\frac{d}{d \tau} i_{1 \rightarrow 2}-\frac{d}{d \tau} i_{2 \rightarrow 1}
\end{aligned}
$$


where $i_{m \rightarrow n}$ and $s_{m \rightarrow n}$ are, respectively, the shares of infectives/susceptibles migrated from node $m$ to node $n$.

Let the population from node 1 migrate between the two nodes, spending a fraction of time $\varepsilon$ in the second node. Suppose that at $\tau=0$, the share $i_{1}$ of infectives is concentrated in node 1 . Under the assumption that the migration is described by linear equations, the share of infectives at node 2 can be described by the convolution with a response function $g(\tau)$ of a linear system. For the problem in hand, it is convenient to write this convolution in the form

$$
i_{1 \rightarrow 2}(\tau)=\frac{d}{d \tau}\left[i_{1}(\tau) * g(\tau)\right]:=\frac{d}{d \tau} \int_{-\infty}^{+\infty} i_{1}\left(\tau^{\prime}\right) g\left(\tau-\tau^{\prime}\right) d \tau^{\prime}
$$

where $g(\tau)=0$ if $\tau<0$ due to the casuality principle. Generally, $i_{1 \rightarrow 2}(\tau)$ converges exponentially to the equilibrium value $\varepsilon i_{1}$ with the characteristic time $\Delta \tau$, for example, from the analogy with a diffusion process it can be

$$
g(\tau)=\varepsilon\left(1-e^{-\tau / \Delta \tau}\right) \theta(\tau)
$$

where $\theta(\tau)$ is the Heaviside unit-step function.

The approximation of weak coupling considered here means that $\varepsilon \ll 1$. Then $i_{m \rightarrow n} \ll 1$ and $s_{m \rightarrow n} \ll 1$.

If those first infected appear in node 1, then the outbreak develops first in this node. Its infectious individuals migrating to node 2 trigger the outbreak there with a certain delay. A node is sensitive to a small amount of infectives only before the outbreak, becoming almost insensitive during and after it. Therefore, we can neglect transport terms in (3.1)-(3.2) as they do not change the essential epidemic dynamics in node 1 . For the approximation of weak coupling, we can also neglect transport terms $i_{2 \rightarrow 1}, s_{2 \rightarrow 1}$ in (3.3)-(3.4). The small term $s_{1 \rightarrow 2}$ in (3.3) also does not impact on the solution of the equation and can be dropped. Thus, in the approximation of weak coupling $(\varepsilon \ll 1)$ and when the outbreak is first initiated in node 1 , the only essential transport term is $i_{1 \rightarrow 2}$ in (3.4) which accounts for infectives migrating from node 1 to node 2 and triggering the outbreak there.

Here, we also restrict our consideration to an approximation of fast migration, when the characteristic time of the migration process $\Delta \tau$ is much smaller than $1 / \lambda_{0}$, i.e. the characteristic time of the epidemic growth on its linear stage

$$
\Delta \tau \ll 1 / \lambda_{0}
$$

In this approximation we can write

$$
g(\tau) \approx \varepsilon \theta(\tau) \text { and } \quad i_{1 \rightarrow 2}(\tau) \approx \varepsilon i_{1}(\tau)
$$

and equations (3.1)-(3.4) coincide with (2.2)-(2.3) whereas equations (3.3)-(3.4) take the form

$$
\begin{aligned}
\frac{d}{d \tau} s_{2} & =-\rho s_{2} i_{2} \\
\frac{d}{d \tau} i_{2} & =\left(\rho s_{2}-1\right) i_{2}+\varepsilon \frac{d}{d \tau} i_{1} .
\end{aligned}
$$


Note that the model (3.9)-(3.10) is different from the well-known model of simple epidemics in interacting groups (see [5], § 2.2) where interaction terms appear without the time derivative. In the fast migration approximation, if the epidemic dynamics are temporarily neglected (spread between individuals and removal are switched off), equations (3.9)-(3.10) imply that $i_{2} \equiv i_{1 \rightarrow 2}=\varepsilon i_{1}$, i.e. the migration provides the only source of infectives in node 2 .

Analogous equations can be derived for many interacting nodes with the external forces, $(d / d \tau) i_{m \rightarrow n}, n, m=1,2, \ldots, m \neq n$, proportional to the rate of change of the number of infectives arriving from other nodes coupled with the $n$th node. They can also describe networks of interacting nodes of different topology (e.g. [10, 17], etc); although in this work we consider the simplest network topology only.

\section{One-dimensional lattice of SIR models}

Consider a lattice of interacting identical SIR nodes. Let the dynamics in the first node $(n=1)$ be described by (2.2)-(2.3) with initial conditions (2.4), and the dynamics in all subsequent nodes ( $n=2,3, \ldots)$ by (3.9)-(3.10) which, assuming fast migration, take the form

$$
\begin{aligned}
\frac{d}{d \tau} s_{n} & =-\rho s_{n} i_{n} \\
\frac{d}{d \tau} i_{n} & =\left(\rho s_{n}-1\right) i_{n}+\frac{d}{d \tau} i_{n-1 \rightarrow n}
\end{aligned}
$$

with $i_{n-1 \rightarrow n}=\varepsilon i_{n-1}$.

Numerical integration of the ODEs (4.1)-(4.2) with different $\rho, \varepsilon$ and $i_{0}$ (see Figure 3) shows

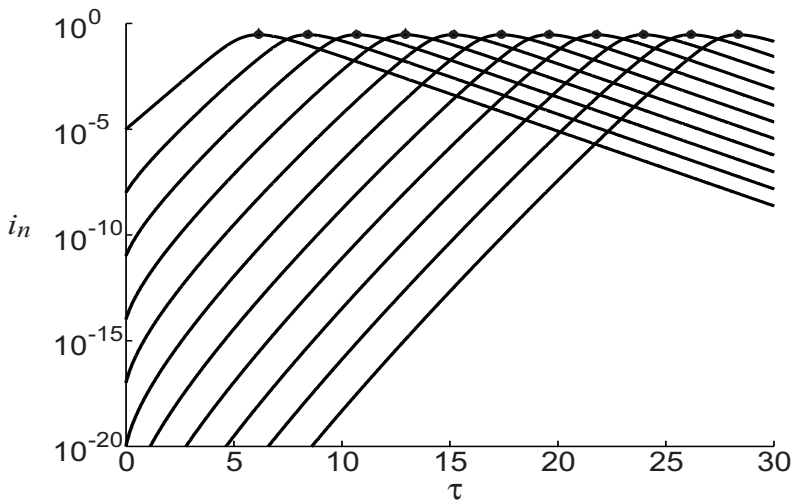

Figure 3: Numerical simulation of lattice with coupled SIR nodes (first 10 nodes) for $\rho=3$ and for $\varepsilon=10^{-3}$.

that the time difference $T_{n}$ between outbreaks in the $(n-1)$ th and $n$th nodes tends to a constant $T(\rho, \varepsilon)$ (see Figure 4). The period of initial growth of $T_{n}$ is more clearly visible in the logarithmical scale (see Figure 4, curve 2). The solution $i_{n}(\tau), s_{n}(\tau)$ for $n \gg 1$ almost replicates the solution 


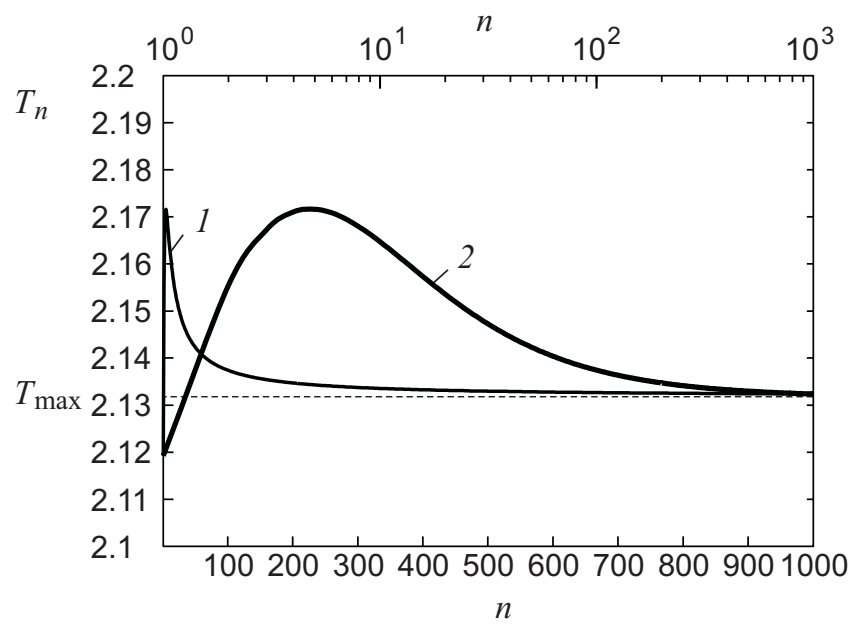

Figure 4: Time interval $T_{n}$ between outbreaks in two neighbouring nodes versus $n$ for $\rho=3$ and $\varepsilon=10^{-3}$ for linear (curve 1 ) and logarithmic (curve 2) $n$-scale. The approximation (4.11) is shown by the dashed line.

$i_{n-1}(\tau), s_{n-1}(\tau)$ in shape, with a delay $T$

$$
i_{n}(\tau) \cong i_{n-1}(\tau+T), \quad s_{n}(\tau) \cong s_{n-1}(\tau+T) .
$$

Thus the numerical integration shows that for $n \rightarrow \infty$ the solution tends to the universal one delayed in time by $n T: i_{n}(\tau) \rightarrow i^{\operatorname{tr}}(\tau-n T)$. This indicates that a type of travelling non-linear wave propagates with the velocity $c=T^{-1}$ in the discretized lattice of individual SIR nodes with unit distances between nodes, whereas the variable $n$ plays the role of a spatial coordinate. Substituting the solution of the form $s_{n}(\tau)=s^{\operatorname{tr}}(\tau-n T), i_{n}(\tau)=i^{\operatorname{tr}}(\tau-n T)$ into (4.1)-(4.2), we obtain a system of two equations

$$
\begin{aligned}
\frac{d}{d \tau} s^{\operatorname{tr}} & =-\rho s^{\operatorname{tr}} i^{\operatorname{tr}} \\
\frac{d}{d \tau} i^{\operatorname{tr}} & =\left(\rho s^{\operatorname{tr}}-1\right) i^{\operatorname{tr}}+\varepsilon \frac{d}{d \tau} i^{\operatorname{tr}}(\tau+T) .
\end{aligned}
$$

Its solution must satisfy the initial conditions

$$
s^{\operatorname{tr}}(-\infty)=1, \quad i^{\operatorname{tr}}(-\infty)=0 .
$$

Here $T=T(\rho, \varepsilon)$ is an eigenvalue of the problem (4.4)-(4.6). As the equations are invariant with respect to a shift in time, we fix $\tau=0$ as an instant when $i^{\text {tr }}$ is maximal.

As the solution $i^{\text {tr }}$ decays for $\tau>0$, the second terms on the r.h.s. of (4.5) are essential only for $\tau<-T$. Hence, for $\tau>-T$ the solution is close to the solution for the individual node considered above. In the limit $\varepsilon \rightarrow 0$ it tends to the limiting solution $i^{\text {lim }}$ in (2.9). Numerical simulation confirms that the travelling wave and limiting solution differ only for $\tau<-T$ (see 


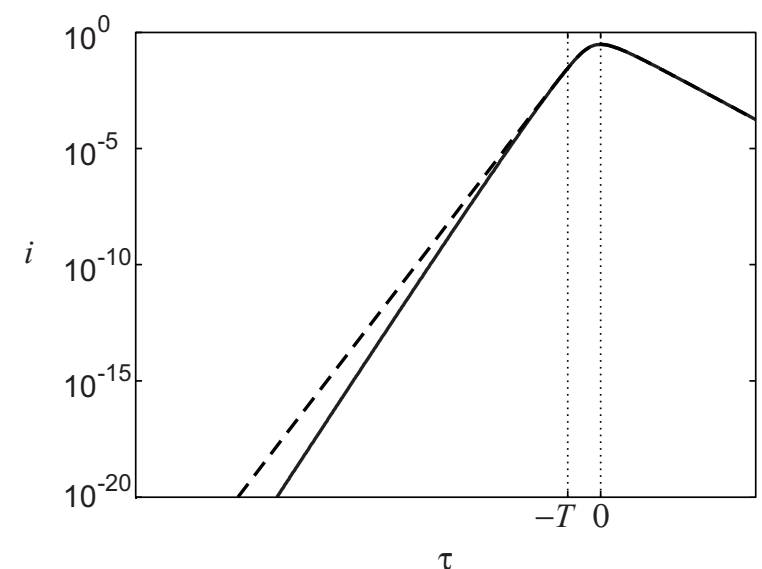

a

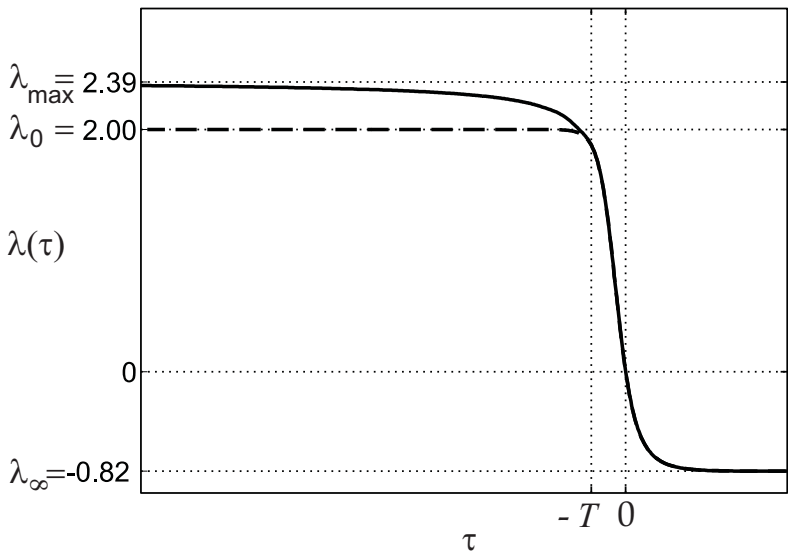

$\mathrm{b}$

Figure 5: Traveling wave $i^{\operatorname{tr}}(\tau ; \rho, \varepsilon)$ (solid) and limiting solution $i^{\lim }(\tau ; \rho)$ (dashed) versus time for $\rho=3$ and $\varepsilon=10^{-3}$ (a). Plot of the growth rate over time, $\lambda(\tau)=(d / d \tau) \ln (i)$ (b).

Figure 5). For $\tau<-T$ the value $s(\tau)$ differs negligibly from 1, and the equation (4.5) can be well approximated by a linear one

$$
\frac{d}{d \tau} i^{\operatorname{tr}}=\lambda_{0} i^{\operatorname{tr}}+\varepsilon \frac{d}{d \tau} i^{\operatorname{tr}}(\tau+T)
$$

Here the solution of (4.7) has an exponential form $i^{\operatorname{tr}}=\exp \{\lambda \tau\}$ where the parameter $\lambda$ is related to the eigenvalue $T$ of the non-linear problem

$$
\lambda=\lambda_{0}+\varepsilon \lambda e^{\lambda T} .
$$

A general approach described $[22,16]$ can be applied to find $T$, and provides the results well supported by numerical evidence. More precisely, in some non-linear systems, the velocity of a travelling wave corresponds to the lowest value taken over a range of wavenumbers for the linearized equation. This should be true for a system in which non-linearity results in slowing down the wave. In fact, the velocity of travelling wave is proportional to the growth rate $\lambda_{0}$ of infinitesimal solution (see below). The higher the wave amplitude $i$, the smaller is the share of susceptibles $s$, hence the smaller is the growth rate which is $\lambda=\rho s-1<\lambda_{0}$. Therefore we find the smallest speed of propagation (greatest $T$ ) for the linear solution and compare it with the results of numerical simulation.

Substituting the plane wave solution of the form

$$
i^{\operatorname{tr}}(\tau, n)=\exp (\lambda \tau-n T)
$$

to (4.1)-(4.2) and neglecting non-linear terms there, we obtain the characteristic equation (cf. (4.8))

$$
L(\lambda, T):=\lambda-\lambda_{0}-\varepsilon \lambda e^{\lambda T}=0 .
$$


Solving the system of equations $L(\lambda, T)=0, L_{\lambda}(\lambda, T)=0$ where $L_{\lambda}=\partial L / \partial \lambda$, we obtain explicit formulas for the maximal value of $T$ and express the correspondent value of $\lambda$ in terms of the Lambert function

$$
T_{\max }=\frac{1}{\lambda_{0}} \frac{\left(W_{0}-1\right)^{2}}{W_{0}}, \quad \lambda_{\max }=\lambda_{0} \frac{W_{0}}{W_{0}-1} .
$$

Here $W_{0}=W_{0}(e / \varepsilon)$ is the main branch of the Lambert function, $e$ is Euler's constant. Dependence of $\lambda_{0} T$ on $\varepsilon$ is plotted in Figure 6.

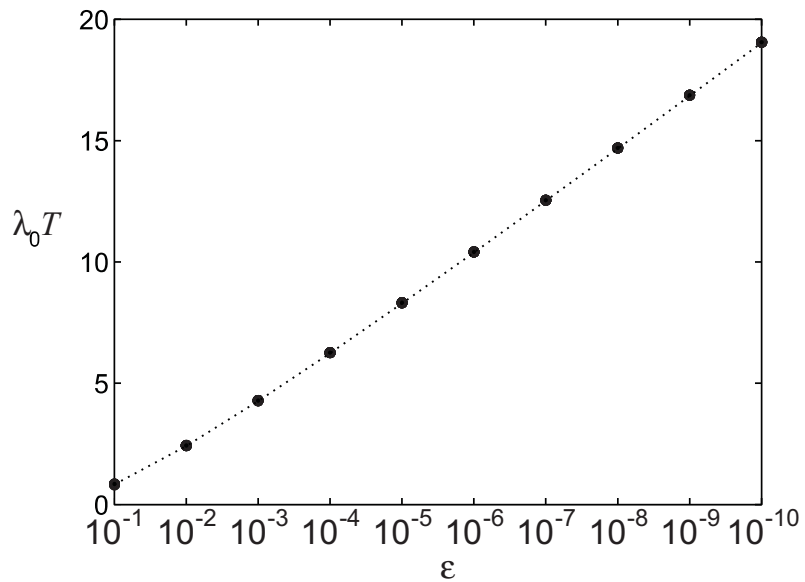

Figure 6: Delay time $\lambda_{0} T$ versus $\varepsilon$ for $\rho=2,3, \ldots, 10$. Circles indicate numerical results. In this scale, all circles for different $\rho$ and the same $\varepsilon$ are merged. Dashed line is drawn by use of (4.11).

The solution has the following expansion for small $\varepsilon$

$$
T_{\max } \approx \frac{1}{\lambda_{0}}\left[\ln \frac{1}{\varepsilon}-\ln \ln \frac{1}{\varepsilon}-1\right] \quad \text { if } \varepsilon \ll 1 .
$$

This converges slowly as an expansion with respect to $\left(\ln \ln \left(\varepsilon^{-1}\right)\right)^{k}$ whereas the Lambert function can be computed easily by fast converging iterations like $W^{(k+1)}(x)=\ln x-\ln W^{(k)}(x)$.

Numerical integration shows that the computed outbreak time difference $T_{n}$ is very close to $T_{\max }$ for sufficiently large $n$ but noticeably exceeds it when $n=O(10)$ (see Figure 4).

Figure $5 \mathrm{~b}$ presents a plot of the current growth/decay rate of $i$

$$
\lambda(\tau)=\frac{d \ln (i)}{d \tau}
$$

The limiting solution $\lambda(\tau)$ passes from the value $\lambda_{0}$ to $\lambda_{\infty}$, and the travelling wave $\lambda(\tau)$ is close to $\lambda^{\text {th }}$ if $\tau$ is small enough. For $\rho=3$ and $\varepsilon=10^{-3}$ we obtain $\lambda^{\text {th }} \approx 2.39, \lambda_{0}=2, \lambda_{\infty} \approx-0.82$. These asymptotes are shown by horizontal dotted lines. 


\section{Lattice with randomized interaction coefficients}

Finally, we consider a lattice of SIR nodes with the same reproduction numbers $\rho$ for every node but randomized interaction coefficients $\varepsilon_{n}$, i.e the lattice is described by equations

$$
\begin{aligned}
\frac{d}{d \tau} s_{n} & =-\rho s_{n} i_{n} \\
\frac{d}{d \tau} i_{n} & =\left(\rho s_{n}-1\right) i_{n}+\varepsilon_{n} \frac{d}{d \tau} i_{n-1} .
\end{aligned}
$$

Let all $\varepsilon_{n}$ be statistically independent, positive, with the mean $\bar{\varepsilon}$ and variance $\sigma_{\varepsilon}^{2}$. We consider the simplest model, with coefficients $\varepsilon_{n}$ having the log-normal distribution, i.e. $\ln \varepsilon_{n}$ have a normal distribution with mean $\mu$ and variance $\sigma^{2}$. Then $T_{n}$ are also normally distributed in the main term of its asymptotic, see (4.12). Values $\bar{\varepsilon}$ and $\sigma_{\varepsilon}$ can be determined from the following relations (e.g. [21])

$$
\bar{\varepsilon}=e^{\mu+\sigma^{2} / 2}, \quad \sigma_{\varepsilon}^{2}=\bar{\varepsilon}^{2}\left(e^{\sigma^{2}}-1\right) .
$$

Resolving (5.3), we find $\mu$ and $\sigma$

$$
\mu=\ln \bar{\varepsilon}-\frac{1}{2} \ln (1+v), \quad \sigma^{2}=\ln (1+v)
$$

where $v=\sigma_{\varepsilon}^{2} / \bar{\varepsilon}^{2}$ is the variance normalized by the square of the mean value. An example of numerical simulation of this model is shown in Figure 7.

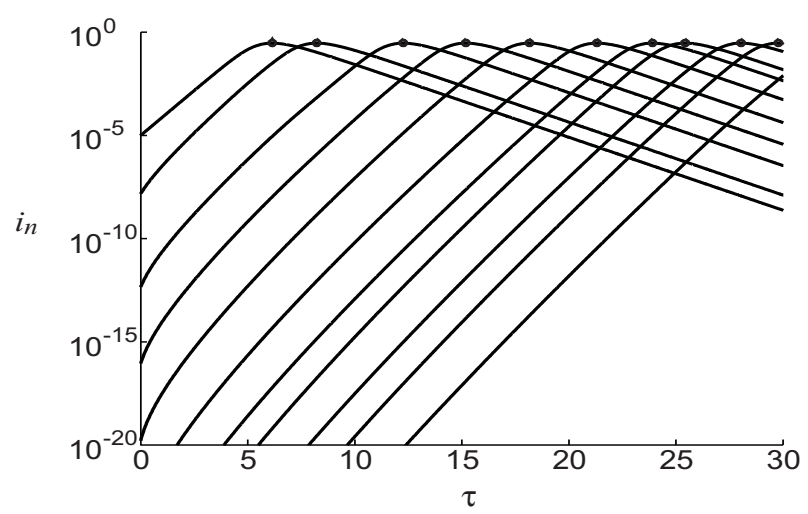

Figure 7: Numerical simulation of lattice with coupled SIR nodes (first 10 nodes) for $\rho=3$ and for randomized $\varepsilon_{n}$ with $\bar{\varepsilon}=10^{-3}$ and $\left(\sigma_{\varepsilon} / \bar{\varepsilon}\right)^{2}=2$.

Assume that in the case $\bar{\varepsilon} \ll 1$ and $\sigma_{\varepsilon} \ll \bar{\varepsilon}$ all time intervals $T_{n}$ between outbreaks in the $(n-1)$ th and $n$th nodes are described by (4.11), i.e.

$$
\lambda_{0} T_{n} \approx \frac{\left(W_{0}\left(e / \varepsilon_{n}\right)-1\right)^{2}}{W_{0}\left(e / \varepsilon_{n}\right)} .
$$


Assumption (5.5) agrees well with numerical simulation and implies that the mean time $\bar{T}$ interval can be calculated via the integral

$$
\lambda_{0} \bar{T}=\int_{0}^{\infty} \frac{\left(W_{0}(e / \varepsilon)-1\right)^{2}}{W_{0}(e / \varepsilon)} f(\varepsilon) d \varepsilon, \quad f(\varepsilon)=\frac{e^{-\frac{(\ln \varepsilon-\mu)^{2}}{2 \sigma^{2}}}}{\sqrt{2 \pi} \sigma \varepsilon} .
$$

Here $f(\varepsilon)$ is the probability density of the log-normal distribution. The substitution $\varepsilon=e^{\mu+\sigma x}=$ $\bar{\varepsilon} e^{\sigma x-\sigma^{2} / 2}$ in (5.6) gives

$$
\lambda_{0} \bar{T}=\frac{1}{\sqrt{2 \pi}} \int_{-\infty}^{+\infty} \frac{\left[W_{0}\left(\frac{e^{1-\sigma x+\sigma^{2} / 2}}{\bar{\varepsilon}}\right)-1\right]^{2}}{W_{0}\left(\frac{e^{1-\sigma x+\sigma^{2} / 2}}{\bar{\varepsilon}}\right)} e^{-\frac{x^{2}}{2}} d x .
$$

Now we expand the integrand into series with respect to $\sigma$ and integrate it term-by-term. Terms with odd powers of $\sigma$ vanish after integration. The first two terms approximate (5.7) very well even for $\sigma$ exceeding unity

$$
\lambda_{0} \bar{T}=\frac{\left(W_{0}-1\right)^{2}}{W_{0}}+\sigma^{2} \frac{W_{0}\left(W_{0}^{2}+2\right)}{8\left(W_{0}+1\right)^{5}}+O\left(\sigma^{4}\right) .
$$

Here $W_{0}=W_{0}(e / \bar{\varepsilon})$. (See Figure 8: closed circles are fitted well by the dotted line).

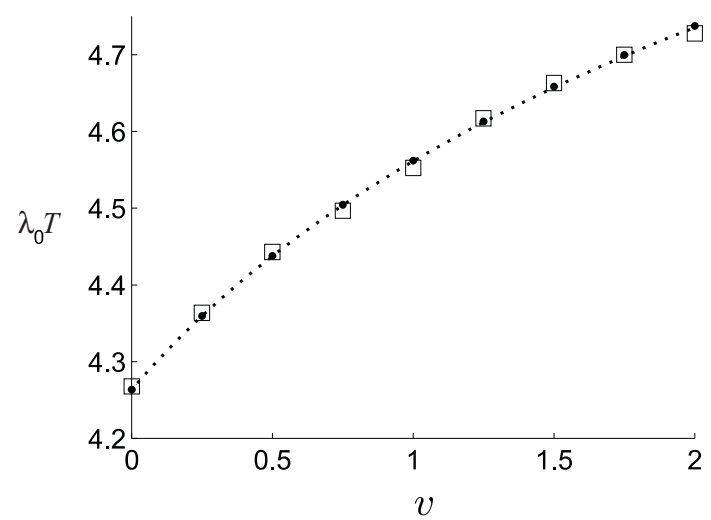

$\mathrm{a}$

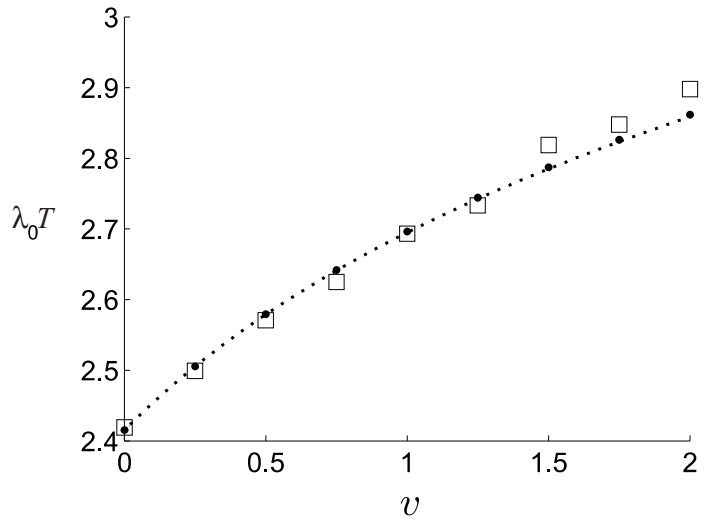

b

Figure 8: Dependence of $\lambda_{0} \bar{T}$ on $v=\left(\sigma_{\varepsilon} / \bar{\varepsilon}\right)^{2}$ for $\rho=3, \bar{\varepsilon}=10^{-3}$ (a) and $\rho=5, \bar{\varepsilon}=10^{-2}$ (b). Numerical results are indicated by open squares. Dotted line indicates the approximation (5.8), closed circles indicate the direct numerical integration of (5.7).

In numerical simulations for a lattice of 401 nodes, the last 200 outbreak intervals $T_{n}$ were taken for averaging. We performed 50 realizations for every $v$, thus every mean value $T_{n}$ was averaged by $10^{4}$ independent values. The results are plotted in Figure 8 . Note that the above assumption fits surprisingly well for rather large values of the variance: $\sigma_{\varepsilon}>\bar{\varepsilon}$. 


\section{Limits of applicability for the approximations}

Here we apply the general approach developed in $([22,16])$ to different modifications of our basic model and compare how these modelling assumptions are reflected in changes to the predicted epidemic speed.

First we consider all the interaction terms between neighbouring lattice nodes

$$
\begin{aligned}
(\partial / \partial \tau) s_{n} & =-\rho i_{n} s_{n}+(\partial / \partial \tau)\left[s_{n-1 \rightarrow n}+s_{n+1 \rightarrow n}-s_{n \rightarrow n-1}-s_{n \rightarrow n+1}\right] \\
(\partial / \partial \tau) i_{n} & =\left(\rho s_{n}-1\right) i_{n}+(\partial / \partial \tau)\left[i_{n-1 \rightarrow n}+i_{n+1 \rightarrow n}-i_{n \rightarrow n-1}-i_{n \rightarrow n+1}\right] .
\end{aligned}
$$

In the linear approximation the equation for $i_{n}$ is independent of $s_{n}$. The linearized equation (6.2) in the fast migration approximation reads as

$$
(\partial / \partial \tau) i_{n}=\lambda_{0} i_{n}+\varepsilon(\partial / \partial \tau)\left[i_{n-1}+i_{n+1}-2 i_{n}\right] .
$$

Substituting the solution in the form $i_{n}(\tau)=e^{\lambda(\tau-n T)}$, we obtain the following characteristic function

$$
L(\lambda, T)=\lambda-\lambda_{0}-\varepsilon \lambda\left[e^{\lambda T}+e^{-\lambda T}-2\right] .
$$

We solve equations $L(\lambda, T)=0, L_{\lambda}(\lambda, T)=0$ numerically (as they have no solution in a closed form) and compare with the expressions (4.11) above. The results for comparison are given in Table 1 for $\varepsilon=10^{-1}, 10^{-2}, 10^{-3}$. Note that an error of neglecting the transport terms is $O(\varepsilon)$. This error is mainly related to neglecting (outgoing) transport terms $i_{n \rightarrow n \pm 1}$ which decreases the epidemic growth. Thus, the reduced form of equations (4.1)-(4.2) is justified in the approximation of weak coupling.

Table 1: Comparison of $T_{\max }$ for different approximations

\begin{tabular}{cccccc}
\hline$\varepsilon$ & $\lambda_{0} T_{\max },(6.4)$ & $\lambda_{0} T_{\max },(4.11)$ & error, $\%$ & $\lambda_{0} T_{\max },(6.8)$ & error, \% \\
\hline $10^{-3}$ & 4.274 & 4.263 & 0.2 & 12.17 & 185 \\
$10^{-2}$ & 2.478 & 2.415 & 2.5 & 3.849 & 60 \\
$10^{-1}$ & 1.097 & 0.833 & 24 & 1.217 & 10 \\
\hline
\end{tabular}

Next we compare the results for the epidemic speed in discrete and continuous spatial models. For this, we introduce the spatial coordinate $x$ instead of $n$

$$
i_{n}(\tau)=i(x, \tau), i_{n+1}(\tau)=i(x+\Delta x, \tau)
$$

and, similarly, for $s_{n}$. Here $\Delta x$ is the distance between nodes (later on we set it to unity). Then we can write

$$
i_{n \pm 1}(\tau)=i(x \pm \Delta x, \tau)=i(x, \tau) \pm \Delta x \frac{\partial}{\partial x} i(x, \tau)+\frac{(\Delta x)^{2}}{2} \frac{\partial^{2}}{\partial x^{2}} i(x, \tau)+O\left((\Delta x)^{3}\right) .
$$


Substituting (6.5) into (6.3), neglecting terms $O\left((\Delta x)^{3}\right)$ and setting $\Delta x=1$, we obtain a linear PDE of the form

$$
\frac{\partial i}{\partial \tau}=\lambda_{0} i+\varepsilon \frac{\partial^{3} i}{\partial^{2} x \partial \tau} .
$$

Substituting the solution of the form $i=e^{\lambda(\tau-x T)}$ into (6.6), we obtain the function

$$
L(\lambda, T)=\lambda-\lambda_{0}-\varepsilon \lambda^{3} T^{2} .
$$

Note that the same equation emerges by expanding $e^{ \pm \lambda T}$ in series with respect to small $\lambda T$ in (6.4). Solving $L(\lambda, T)=0, L_{\lambda}(\lambda, T)=0$ we obtain

$$
T_{\max }=\frac{2}{3 \lambda_{0}} \frac{1}{\sqrt{3 \varepsilon}}, \quad \lambda_{\max }=\frac{3 \lambda_{0}}{2} .
$$

Note that the asymptotic behaviour of $T_{\max }$ for small $\varepsilon$ is different: $\varepsilon^{-1 / 2}$ in (6.8) in contrast with $\ln \left(\varepsilon^{-1}\right)$ in (4.12). The results for comparison are also given in Table 1.

We conclude that for $\varepsilon \leq 10^{-2}$ the weak coupling approximation gives good accuracy, whereas the error for the continuous model becomes large. If $\varepsilon$ approaches 0.1 , the continuous approximation gives more accurate result for epidemic speed than the small coupling approximation although the latter still has the reasonable accuracy $O(\varepsilon)$.

It worth to mention that if in discrete model we account for the outgoing migration terms $\left(i_{n \rightarrow n \pm 1}\right)$ but neglect the incoming migration from a forward node $\left(i_{n+1 \rightarrow n}\right)$ (the characteristic function in this case has the form $L(\lambda, T)=\lambda-\lambda_{0}-\varepsilon\left(e^{\lambda T}-2\right)$ ) then the system $L=0, L_{\lambda}=0$ has an explicit solution expressed via the Lambert function of somewhat different argument

$$
T=\frac{\left(W_{0}-1\right)\left[(1+2 \varepsilon) W_{0}-1\right]}{W_{0}}, \quad \lambda=\frac{W_{0}}{\left(W_{0}-1\right)(1+2 \varepsilon)}, \quad W_{0}=W_{0}\left[\frac{(1+2 \varepsilon) e}{\varepsilon}\right] .
$$

Then the relative errors in $T$ compared to the numerical solution of (6.4) are $10^{-5}$, $5 \times 10^{-4}, 3 \times 10^{-2}$ for $\varepsilon=10^{-3}, 10^{-2}, 10^{-1}$, respectively. This is the most accurate explicit formula for $T$.

Also based on approach described in $[22,16]$, we can estimate the accuracy of the fast migration approximation. The linearized equation in this case takes the form

$$
\frac{\partial}{\partial \tau} i_{n}(\tau)=\lambda_{0} i_{n}(\tau)+\frac{\partial^{2}}{\partial \tau^{2}} \int_{-\infty}^{\tau} i_{n-1}\left(\tau^{\prime}\right) g\left(\tau-\tau^{\prime}\right) d \tau^{\prime}
$$

Here we use the kernel $g(\tau)$ described by (3.6). Substituting $i_{n}=e^{\lambda(\tau-n T)}$, we obtain the characteristic function

$$
L(\lambda, T)=\lambda-\lambda_{0}-\varepsilon \frac{\lambda e^{\lambda T}}{\lambda \Delta \tau+1} .
$$

The results of numerical solutions for $L(\lambda, T)=0, L_{\lambda}(\lambda, T)=0$ are plotted in Figure 9. Observe that for small $\varepsilon$ the increment $T(\varepsilon, \Delta \tau)-T(\varepsilon, 0)$ depends slightly on $\varepsilon$ (see Figure 9a). This leads to a smaller relative change of the epidemic speed for small $\varepsilon$ (see Figure 9b). 


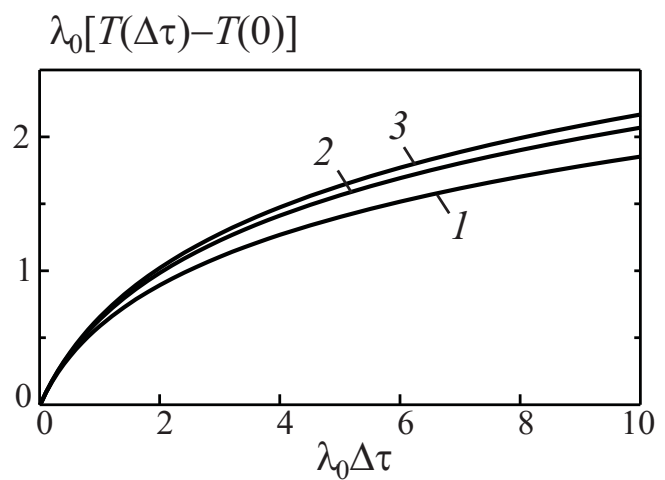

a

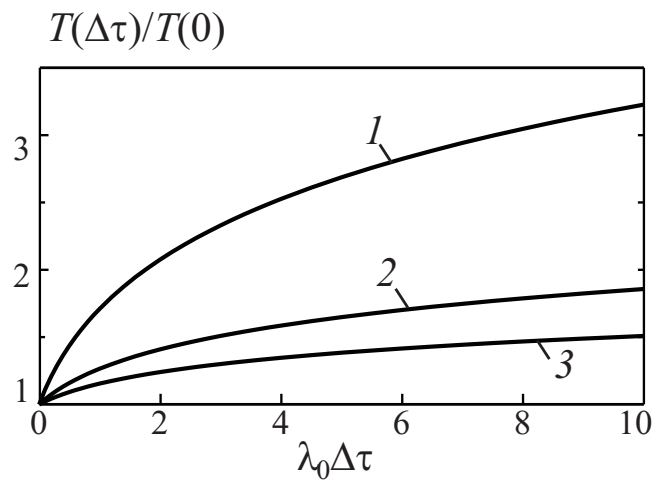

$\mathrm{b}$

Figure 9: Change of $T$ cause by finite migration characteristic time $\Delta \tau$ : absolute (a) and relative (b) increment of $T(\tau)$ for $\varepsilon=10^{-1}$ (curve 1 ), $10^{-2}$ (curve 2) and $10^{-3}$ (curve 3 ).

\section{Discussion}

A 1D lattice of interacting SIR epidemic models is considered in the approximation of weak interactions and fast migration. Numerical simulations confirm that over a wide parameter range $\left(\varepsilon=10^{-10} \div 10^{-1}, \rho=2 \div 10\right)$, the solution tends to an universal one which behaves as a travelling wave preserving its shape and speed. In the framework of this model, a simple explicit formula is obtained for the speed of the epidemic spread in a lattice with either constant or random interaction coefficients. Compared with numerical simulations, the solutions for the randomized case demonstrate surprisingly good accuracy over a wide range of applicability, i.e. when the variance of the coefficient exceeds the square of its mean value. In the weak coupling approximation the developing outbreak in every node of the lattice is well approximated by the limiting solution obtained for a single SIR model.

A similar analysis is performed for the finite characteristic migration time $\Delta \tau$, and responses of the form given in (3.5)-(3.6). The qualitative behaviour of the travelling waves remains the same, with the speed reduced compared to the approximation of fast migration. The shape of the travelling wave around the outbreak will presumably remain the same and be well approximated by the limiting solution. The numerical simulation of a lattice with the finite $\Delta \tau$ and random coupling parameters will be a subject of future work.

Note that the results of this paper can be straightforwardly extended to a response function of form $g(\tau)=\varepsilon \theta(\tau-\delta)$. We simply add $\delta_{n}$ to $T_{n}$ obtained numerically (see Figure 4 ) or theoretically (see (4.11)), i.e. $T_{n}^{\delta}=T_{n}+\delta_{n}$ where $\delta_{n}$ is the migration delay between the $n$th and $(n+1)$ th nodes. In the case of a Gaussian distribution of coupling coefficients, the distribution of $T_{n}^{\delta}$ remains Gaussian in the main term of the approximation with the mean value of $T_{n}^{\delta}$ being the sum of $\bar{T}$ (5.8) and the mean of $\delta_{n}$.

In the present work, we concentrate on an asymptotic case when the number of introduced infectives is small compared to the number of cases generated during the outbreak. In the lattice 
model, this is equivalent to small interaction coefficients (weak coupling approximation). In this approximation, the outbreak time (in a single SIR) and the time between outbreaks in neighbouring nodes are logarithmically large: see (2.12) for a single SIR model and (4.12) for the lattice. The numerical solutions in Section 6 (see Table 1) demonstrate that an approximation of finite differences by derivatives, i.e. the models of reaction-diffusion PDEs [18, 8, 14, 6, 3] lead to significant errors in the value of propagation speed, and thus a discrete spatial model is essential for the analysis (cf. $[19,12])$. Clearly, the problem of determining whether the discrete or continuous nature of the phenomena should be considered is rather serious (see discussion in the paper 'The importance of being discrete and spatial' [19]). In contrast to this paper we do not consider a population of discrete individuals, however we show that the step from continuous media to discreteness in space can itself lead to different predictions as demonstrated here.

Our formulae for the speed and shape of a travelling wave in a lattice of SIR models potentially provides the means to estimate the interaction coefficients between urban populations in relevant ecological databases. One of the key examples from epidemiology, that links an extensive database to an underlying travelling wave model is based on the records of measles outbreaks in and around London since the 1940s [6]. In the case of the measles data however, it was concluded that the outbreaks in large cities were in reality synchronized by strong linkage, endemic status and a shared seasonal forcing term in the contact rates (due to the school year). In contrast, in a lattice of towns and small population centres, travelling waves could persist if there was local extinction of disease in small centres. The outbreaks in these centres will therefore tend to lag behind the epidemic generated in a larger community until the weak coupling between the towns sparks a new outbreak. We note that our derivation of time to the peak of the outbreak can also provide a simple approximation for the fade-out time of the outbreak (e.g., by defining the extinction time when the number of infected individuals is reduced below 1). If travelling waves in epidemiology are caused by an interaction of weak coupling, and local centre extinction, our model provides a useful analytical framework for investigating the underlying mechanisms of observed travelling waves using long term historical records such as certain measles scenarios, and other directly transmitted infections such as the influenza viruses.

The problems addressed here are characterized by different time scales, $T, \Delta \tau$ and $1 / \lambda_{0}$. A more delicate use of 'separation of scales' phenomena and backward influence will be exploited in forthcoming papers. We also intend to study other types of deterministic or random coupling and other distributions of random coupling constants.

Although a network of the simplest topology is considered here (intuitively it corresponds to a sequence of towns located along a railway or highway), a more general interpretation of the results is required. A 2D system would clearly fit more closely with the geographical spread of real diseases. From a physical viewpoint, the 1D lattice corresponds to a plane wave approximation, which is standard for a cylindrical wave far from the source. We believe that away from a localized source, a cylindrical epidemic follows more and more the pattern described by the 1D model considered in our work. A more specific description of this phenomena will be a subject of future work.

Although very well studied, the SIR model continues to provide analytical insight into the basic mechanisms of epidemic processes. This basic model cannot represent all the features of real-life 
phenomena, it is often found that the more complex simulation-type models are used in the case of specific outbreaks and to plan actual interventions. However, there can be concerns over assessing the validity of complex models, that include many nodes, and how the large number of assumptions and parameter values determine the model output [13]. There is therefore a need for continuous development of analytical models of the spatial evolution of epidemics (e.g. $[2,11])$ to provide a theoretical basis for those used in planning scenarios.

\section{Acknowledgements}

The work was supported by EPSRC Project EP/F014015/1. We would like to thank anonymous referees for helpful comments.

\section{References}

[1] R.M. Anderson, R.M. May. Infectious Disease of Humans: Dynamics and Control. Oxford Univ. Press, Oxford, 1991.

[2] D. Brockmann, L. Hufnagel, T. Geisel. Dynamics of modern epidemics. In SARS: A Case Study in Emerging Infections. Edited by A.R. McLean, R.M. May, J. Pattison, and R.A. Weiss. Oxford Univ. Press, Oxford, 2005.

[3] R.S. Cantrell, C. Cosner. Spatial Ecology via Reaction-Diffusion Models. Mathematical Surveys and Monographs. Wiley, Chichester, 2003.

[4] R.M. Corless, G.H. Gonnet, D.E.G. Hare, D.J. Jeffrey, and D.E. Knuth. On the Lambert W function. Advances in Computational Math., 5 (1996), 329-359.

[5] D.J. Daley, J. Gani. Epidemic Modelling. Cambridge Univ. Press, Cambridge, 1996.

[6] B.T. Grenfell, O.N. Bjørnstad, J. Kappey. Traveling waves and spatial hierarchies in measles epidemics Nature, 414 (2001), No. 13, 716-723.

[7] J.A.P. Heesterbeek. A brief history of $R_{0}$ and a recipe for its calculation. Acta Biotheor., 50 (2002) No. 5, 375-376.

[8] Y. Hosono, B. Ilyas. Traveling waves for a simple diffusive epidemic model. Math. Models \& Methods in Appl. Sciences., 5 (1995), No. 7, 935-966.

[9] R.R. Kao. The role of mathematical modelling in the control of the 2001 FMD epidemic in the UK. Trends in Microbiology, 10 (2002), 279-286.

[10] M.J. Keeling, K.T.D. Eames. Networks and epidemic models. J. R. Soc. Interface, 2 (2005), 295-307. 
[11] E. Kenah, J.M. Robins. Second look at the spread of epidemics on networks. Phys. Rev. E, 76 (2007) No. 3, Pt. 2, 036113.

[12] S.A. Levin, R. Durrett. From Individuals to epidemics Philos. Trans.: Biological Sciences, 351 (1996), 1615-1621.

[13] R.M. May. Uses and abuses of mathematics in biology. Science, 303 (2004), 790-793.

[14] V. Mendez. Epidemic models with an infected-infectious period. Phys. Rev. E., 57 (1998) No. 3, 3622-3624.

[15] D. Mollison, ed. Epidemic Models: Their Structure and Relation to Data, Cambridge Univ. Press, Cambridge, 1995.

[16] D. Mollison. Dependence of Epidemic and Population Velocities on Basic parameters. Math. Biosciences, 107 (1991), 255-287.

[17] C. Moore, M. Newman. Epidemics and percolation in small-world networks. Phys. Rev. E, 61 (2000), 5678-5682.

[18] J.D. Murray. Mathematical Biology. Springer-Verlag. London, 1989.

[19] R. Durrett, S.A. Levin. The importance of being discrete (and spatial), Theor. Pop. Biol. 46 (1994), 363-394.

[20] L. Rass, J. Radcliffe. Spatial deterministic epidemics. Vol. 102 of Mathematical Surveys and Monographs. Amer. Math. Soc. Providence RI, USA, 2003.

[21] Y. Suhov, M. Kelbert. Probability and Statistics by Example. Vol. I. Cambridge Univ. Press, Cambridge, 2005.

[22] F. van den Borsch, J.A.J. Metz, O. Diekmann. The velocity of spatial population expansion, J. Math. Biol., 28 (1990), 529-565.

\section{A Approximations for the outbreak time}

Dividing (2.3) by (2.2) and integrating the obtained equation, taking into account (2.4), we obtain the dependence of $i$ versus $s$ (cf. [18])

$$
i=1-s+\frac{1}{\rho} \ln \frac{s}{s_{0}}
$$

The inverse dependence can be written via the Lambert $W_{k}(x)$ function, i.e. the $k$ th branch of solution of $W \exp (W)=x$ [4] (alternatively called ProductLog in the algebraic package MATHEMATICA)

$$
s=-\frac{1}{\rho} W_{k}\left(-\rho s_{0} e^{\rho(i-1)}\right)
$$


where $k=-1$ for the growing part of the solution and $k=0$ for the decaying part. Setting $i=0$ we obtain an explicit formula for $s_{\infty}$

$$
s_{\infty}=-\frac{1}{\rho} W_{0}\left(-\rho s_{0} e^{-\rho}\right)
$$

which gives (2.8) by setting $s_{0}=1$. Substituting (A1) into (2.2) and integrating $\tau(s)$ in the limits $\left[s_{\max }, s_{0}\right]$, we obtain formula (2.10).

To study the asymptotic behaviour of (2.10) for $i_{0} \rightarrow 0$ we make the substitution $s=1-\xi$

$$
\tau_{\text {outb }} \cong \int_{i_{0}}^{1-\rho^{-1}} \frac{d \xi}{f(\xi)}
$$

where $f(\xi)=(1-\xi)\left(\rho \xi+\ln \left((1-\xi) /\left(1-i_{0}\right)\right)\right)$.

Denote the root of $f(\xi)$ with the minimal absolute value by $\xi_{0}=-i_{0}(\rho-1)^{-1}+O\left(i_{0}^{2}\right)$. Consider the function

$$
F\left(\xi ; i_{0}\right)=\frac{1}{f(\xi)}-\frac{1}{f^{\prime}\left(\xi_{0}\right)\left(\xi-\xi_{0}\right)} .
$$

It is regular in the vicinity of $\xi=0$, and it is easy to show that $F\left(\xi ; i_{0}\right)=F(\xi ; 0)+O\left(i_{0}\right)$, $\forall \xi \in[0,1]$. Hence,

$$
\int_{i_{0}}^{1-\rho^{-1}} F\left(\xi ; i_{0}\right) d \xi=\int_{0}^{1-\rho^{-1}} F(\xi ; 0) d \xi+O\left(i_{0}\right)
$$

Our goal is to represent $\tau_{\text {outb }}$ as a term independent of $i_{0}$ plus a correction

$$
\tau_{\text {outb }} \cong\left[\int_{0}^{1-\rho^{-1}} F(\xi ; 0) d \xi+O\left(i_{0}\right)\right]+\int_{i_{0}}^{1-\rho^{-1}} \frac{d \xi}{f^{\prime}\left(\xi_{0}\right)\left(\xi-\xi_{0}\right)}
$$

To this aim, we estimate the correction represented by the second integral

$$
\int_{i_{0}}^{1-\rho^{-1}} \frac{d \xi}{f^{\prime}\left(\xi_{0}\right)\left(\xi-\xi_{0}\right)}=\frac{1}{\lambda_{0}}\left[\ln \frac{1}{i_{0}}+2 \ln \left(1-\rho^{-1}\right)\right]+O\left(i_{0}\right) .
$$

This argument also yields an expression for constant $C(\rho)$ in (2.12)

$$
C(\rho)=\int_{0}^{1-\rho^{-1}} F(\xi ; 0) d \xi+\frac{2 \ln \left(1-\rho^{-1}\right)}{\lambda_{0}} .
$$

The function $f(\xi)$ vanishes at $\xi=0$ and $\xi=1$. As the integrand in (A3) grows towards the limits of the integration domain, its boundaries are the main contributors, especially the integration near its lower limit $\left(\xi=i_{0}\right)$. Approximating $f(\xi)$ by the second order polynomial

$$
\mathcal{P}_{2}(\xi)=\lambda_{0} \xi(1-\xi)
$$




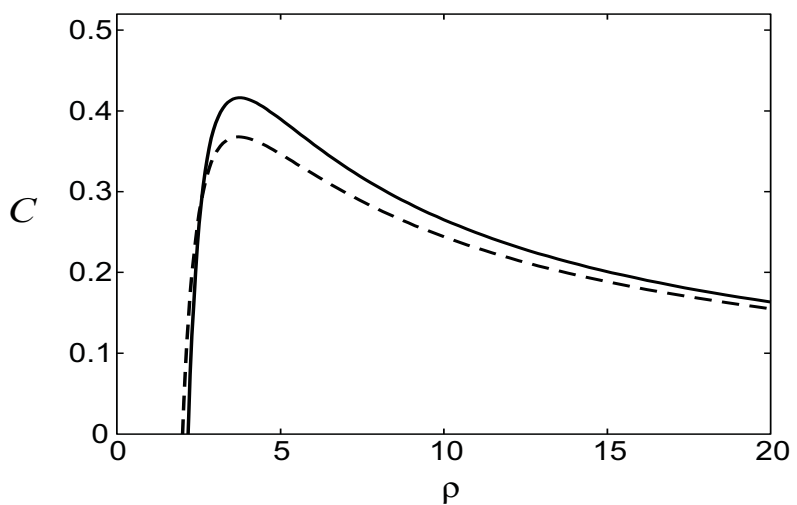

Figure 10: Outbreak time coefficient $C_{0}$ (a) vs $\rho$ : exact—solid, approximation—dashed line.

which vanishes at $\xi=0,1$ and matches the first derivative $f^{\prime}(0)$, and neglecting non-essential terms at $i_{0} \rightarrow 0$, we obtain

$$
C \approx \frac{\ln \left(\lambda_{0}\right)}{\lambda_{0}}
$$

that gives $A_{0} \approx \lambda_{0}$ in accordance with (2.13). Comparison between $C(\rho)$ computed numerically and approximated by (A6) is shown in Figure 10. 